\title{
Sampling quantization analysis and results for FMCW SAR
}

\author{
Adriano Meta*, Peter Hoogeboom ${ }^{\dagger}$ and Leo P. Ligthart ${ }^{\dagger}$ \\ ${ }^{*}$ German Aerospace Center, Microwave and Radar Institute, \\ P.O. Box 1116, 82230 Wessling, Germany, email: adriano.meta@dlr.de \\ ${ }^{\dagger}$ International Research Centre for Telecommunications-transmission and Radar, \\ Delft University of Technology, The Netherlands
}

\begin{abstract}
One of the advantage of Frequency Modulated Continuous Wave (FMCW) radars is the relative low required sampling frequency even when transmitting high bandwidths. This is a consequence of the inherent homodyne configuration of the radar front-end. A simple way to reduce further the data rate is to use few bit per sample. The paper analyzes the effects of low bit sampling in FMCW Synthetic Aperture Radar (SAR) data. A low number of bits used to sample the FMCW deramped signal could produces spurious peaks and intermodulation products of the sinusoidal signal. However, it is shown in the paper that, for typical value of signal to noise ratio in FMCW systems, white noise suppresses the spurious products. Simulation results and verifications on real data collected with the FMCW SAR system built at the Delft University of Technology are presented.
\end{abstract}

\section{INTRODUCTION}

The combination of Frequency Modulated Continuous Wave (FMCW) technology with Synthetic Aperture Radar (SAR) techniques leads to lightweight, cost-effective imaging sensors of high resolution. Airborne FMCW SAR have been successfully built and operated [1], [2]. Such systems can represent a valid alternative to conventional pulse SAR for several applications which require low altitude deployment of the aircraft. One of the advantages of FMCW is the relative low required sampling frequency even when transmitting high bandwidths. This is a consequence of the inherent homodyne configuration of the radar front-end [3], [4]. From a hardware point of view it is therefore easier to add receiving channels in an FMCW system than in a pulse radar. A direct consequence of having more receiving channels is that polarimetric and interferometric data acquisitions can be made with a single pass measurement. Other applications which require multiple channels are i.e. Moving Target Indication and SAR Digital Beam Forming configurations. For the latter, FMCW SAR could represent a good choice for the testing and validation of developed dedicated algorithms.

As said, the amount of data to be acquired and stored with FMCW SAR is relatively small compared to pulse systems because of the deramp-on-receive characteristic; however it can still be high enough to pose problems in the realization of systems using very cheap off-the-shelf components, i.e. cheap PC based components. For instance, sampling 8 channels at 40 $\mathrm{MHz}$ requires the sampling and the storage of $320 \mathrm{M}$ samples per second, continuously during the SAR acquisition. Even if the sampling can be achieved relatively easily, the storage can be problematic for the amount of data per second to be stored continuously. A simple solution is to reduce the number of bits per sample to be stored.

In pulse radars, Block Adaptive Compression (BAQ) techniques are usually used when needed [5]. They divide the range profile in block and then equalize the amplitude variance of the blocks before sampling them with a reduced number of bits. This approach however, is not directly suitable for deramped data because the signal is spread through the whole time sweep duration. In FMCW, however, the fact that the radar is continuously receiving can be exploited to reduce the number of bits directly during the sampling without any additional processing.

The paper confirms that, for typical values of the thermal noise in the FMCW receivers, the quantization noise introduced by a reduced number of bits does not produce spurious peaks associated with the main target response. The consequence of using a reduced number of bits is a higher level of the quantization noise.

\section{THE FMCW RADAR PRINCIPLE}

FMCW is a continuous wave $(\mathrm{CW})$ radar which transmits a frequency modulated (FM) signal [3]. In linear FMCW radars, the used modulation is usually a sawtooth. The ramp is also known as a chirp, see Fig. 1(a). Objects illuminated by the antenna beam scatter part of the transmitted signal back to the radar, where a receiving antenna collects this energy. The travelling time for a target at distance $r$ is given by $\tau=2 \mathrm{r} / \mathrm{c}$ where $c$ is the speed of light. In a homodyne FMCW receiver, the received signal is mixed with a replica of the transmitted waveform and low pass filtered. This process is usually called stretching or deramping. The resulting output is called the beat (or intermediate frequency, or deramped) signal: From Fig. 1(a), it can be seen that the frequency of the beat signal is directly proportional to the target time delay, and hence to the distance. The beat frequency is expressed as:

$$
f_{b}=\frac{B}{P R I} \tau
$$




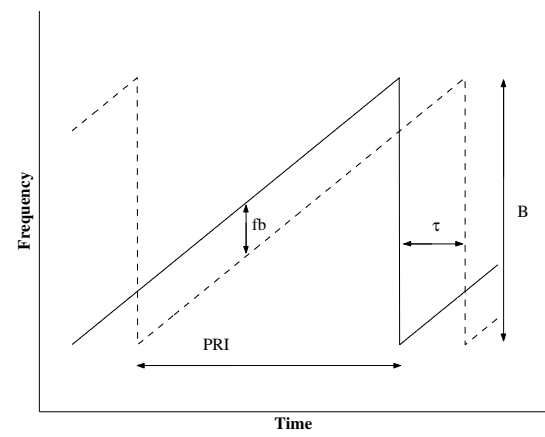

(a)

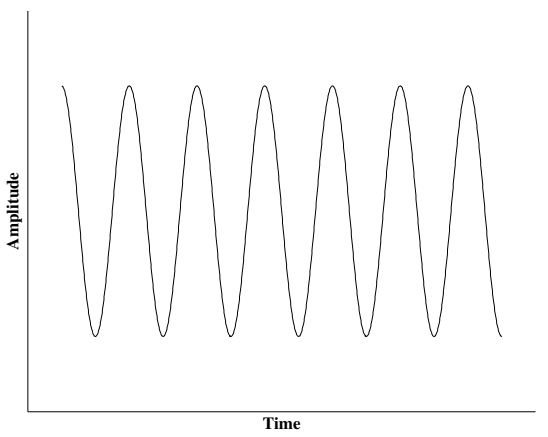

(b)

Fig. 1. Overview of the linear FMCW radar principle. In (a): frequency plot of the chirp signal; the received signal (dashed) is a delay version of the transmitted (solid). In (b): beat signal representation in the time domain. The signal frequency is proportional to the scatterer distance.

where $B$ is the transmitted bandwidth and $P R I$ is the pulse repetition interval.

In order to compress the range response, a Fourier transform is performed on the beat signal (see Fig. 1(b)), making the signal content available in the frequency domain. A practical resulting signal from an FMCW sensor is the superposition of different sinusoidal signals, corresponding to the environment being illuminated by the radar waves.

\section{SPURIOUS QUANTIZATION PEAKS}

Differently from most of conventional pulse radars, the acquisition system of an FMCW sensor is sampling directly the deramped signal, that is a superposition of sinusoids. It is known that in absence of noise, or for high value of signal to noise ratio (SNR), an analog to digital converter introduces harmonics and intermodulation products due to quantization errors. However, the presence of white noise has a linearizing effect on the converter. In order to analyze the effects of a low bit sampling, a sinusoidal signal with $1 \mathrm{~ms}$ duration and sampled at $5 \mathrm{MHz}$ is simulated. Successively white gaussian noise is added, and the resulting signal is sampled with 12 and 2 bits, respectively. The peak to peak sampler range is set to four times the standard deviation of the signal to be sampled. The simulation is repeated with different SNR values: $40 \mathrm{~dB}, 20 \mathrm{~dB}, 0 \mathrm{~dB}$ and $-10 \mathrm{~dB}$. The SNR refers to the deramped signal. Results are shown in Fig. 2, where the Fourier transform is plotted. It can be noticed that the presence of spurious peaks decreases with decreasing SNR, and they already disappear in the $10 \mathrm{~dB}$ SNR example. In fact, good performance of low bit quantization is related to the presence of large additive noise in the raw data. This condition is usually satisfied in airborne FMCW SAR applications. For instance, the SNR of a $10 \mathrm{dBm}^{2}$ point scatterer at $3 \mathrm{~km}$ distance is approximately $0 \mathrm{~dB}$ for an $\mathrm{X}$ band sensor transmitting $1 \mathrm{~W}$ power, with $5 \mathrm{MHz}$ bandwidth noise, and with $5 \mathrm{~dB}$ noise figure.

The quantization noise should be close to the thermal noise level in order to suppress the spurious peaks. This gives some freedom in the choice of the number of bits per sample depending on the sampling frequency and pulse length.

\section{REAL DATA ANALYSIS}

A demonstrator system has been realized at the International Research Centre for Telecommunication and Radar (IRCTR) of the Delft University of Technology using an X-band FMCW front-end. In the autumn of 2005, a flight test campaign was carried out and images were produced, showing the great potential of such a system [1]. FMCW SAR images transmitting up to $500 \mathrm{MHz}$ bandwidth were generated. High resolution images have been obtained using special processing techniques [6] [7] to eliminate the frequency non-linearity problem, typical of low cost FMCW. This method requires a precise phase estimation of the non-linearity directly in the raw data. Therefore analysis of low bit quantization effects on the amplitude and phase have been performed on the real data acquired with the Delft FMCW SAR system. The data were original acquired with 12 bits per sample; however, the raw signal spans only 7 bits. Successively, from the original data set, raw data sampled with 2 bits are extracted, keeping the same peak to peak sampling range. The data are first preprocessed with the non-linearity correction method and then focussed with standard SAR techniques.

The processed images are reported in Fig. 3. It can be noticed that the resolution is not worsened in the 2 bits image. Therefore, the phase estimation of the non-linearity correction algorithm works well on the 2 bits data. On the other hand, an increased noise level can be noticed due to the quantization noise. When compared to the 12 bits image, the dynamic range of the intensity in the 2 bits SAR image is decreased. This effect can be seen in the range profile comparison reported in Fig. 3. However, a dynamic range of more than $30 \mathrm{~dB}$ is still kept when sampling at 2 bits per sample.

An interferogram of the two complex slant range SAR images has been produced in order to check the effects on the phase. Some noise is visible in areas where quantization noise is the limiting factor. In areas where the quantization noise is 

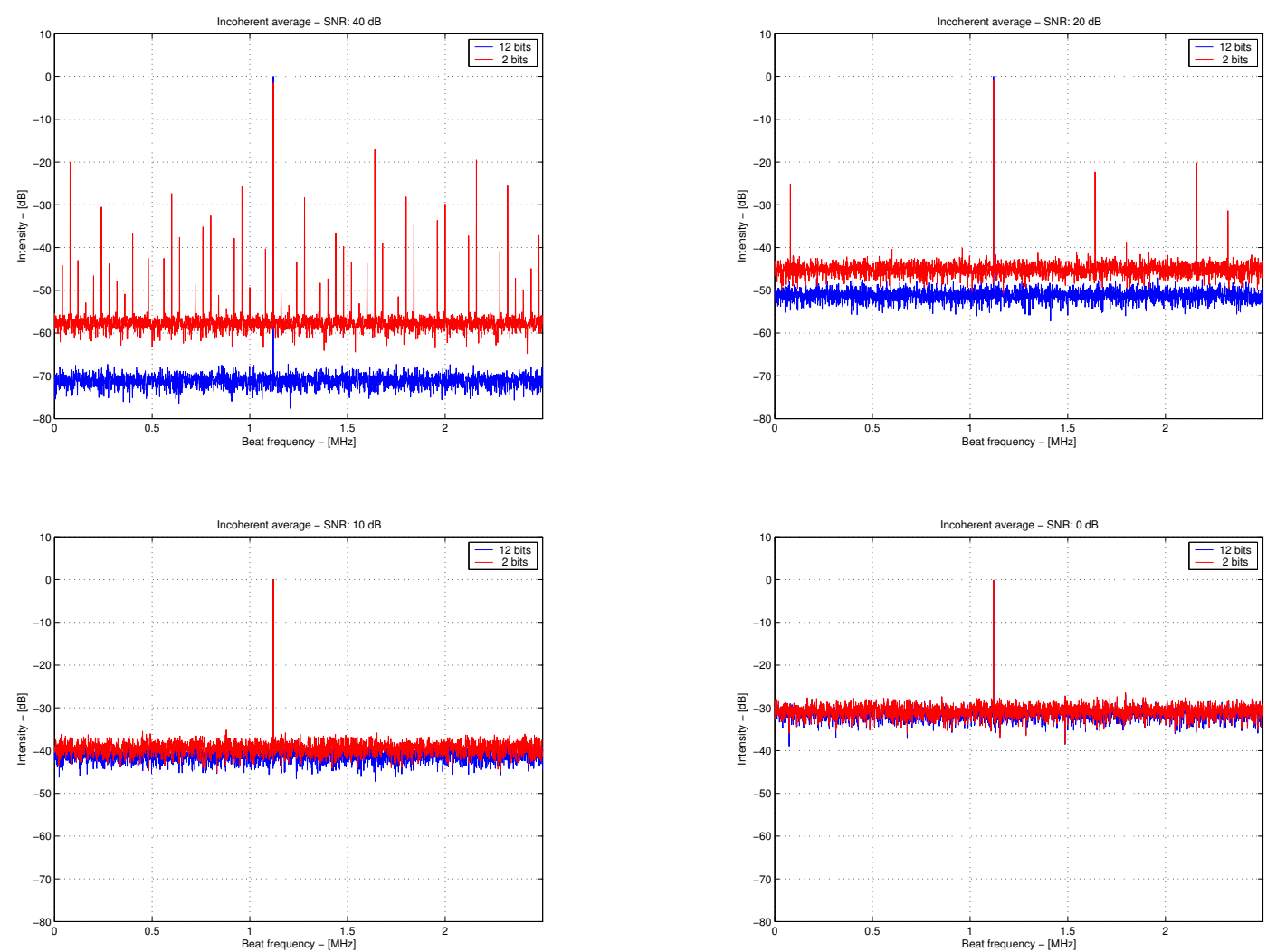

Fig. 2. Simulation results: a $1.12 \mathrm{MHz}$ sinusoid has been generated with different SNR values $(40 \mathrm{~dB}, 20 \mathrm{~dB}, 0 \mathrm{~dB}$ and $-10 \mathrm{~dB})$ and then real sampled with 12 and 2 bits at $5 \mathrm{MHz}$. The SNR refer to the deramped signal.

still belove the signal response, no degradation of the phase is visible. This suggests that polarimetric and interferometric application should not suffer from a sampling with a reduced number of bits, in images with a proper signal to noise ratio.

The results obtained are in line with one bit sampling solutions proposed in [8] [9] and applied to conventional chirp pulse radar.

\section{CONCLUSIONS}

Analyses on a low bit quantization of the deramped FMCW signal have been performed on simulated data and on real data. For typical value of SNR in airborne FMCW SAR application, problem concerning spurious peaks are negligible. Results show that a dynamic range of more than $30 \mathrm{~dB}$ in an intensity SAR image produced with the collected data is still achieved when sampling the FMCW raw data with only 2 bits per sample. Analyses of the single look SAR phase have also shown that the phase is negligibly affected, allowing therefore the use of the produced images for polarimetric and interferometric applications. The real data have been acquired using 12 bits per sample. For the analysis of the paper, the reduced number of bits is extracted directly from the original raw data.

The results of the paper confirm that a multichannel FMCW SAR system can be indeed built using cheap and standard components not only for the front-end part but also for the acquisition and storage system.

\section{REFERENCES}

[1] A. Meta, P. Hakkaart, F. V. der Zwan, P. Hoogeboom, and L. P. Ligthart, "First demonstration of an X-band airborne FMCW SAR," in Proc. European Conf. on Synthetic Aperture Radar EUSAR'06, Dresden, Germany, May 2006.

[2] M. Edrich, "Design Overview and Flight Test Results of the Miniaturised SAR Sensor MISAR," in Proc. EuRAD'04, Amsterdam, The Netherlands, Oct. 2004, pp. 205-208.

[3] M. I. Skolnik, Introduction to Radar Systems. McGraw-Hill, Inc., 1980.

[4] A. G. Stove, "Linear FM-CW Radar Techniques," IEE Proc. Part F Radar Signal Process., vol. 139, no. 5, pp. 343-350, Oct. 1992.

[5] R. Kwok and W. T. K. Johnson, "Block adaptive quantization of Magellan SAR data," IEEE Trans. Geosci. Remote Sensing, vol. 27, no. 4, pp. 375383, 1989.

[6] A. Meta, P. Hoogeboom, and L. P. Ligthart, "Range non-linearities correction in FMCW SAR," in Proc. IEEE Int. Geoscience and Remote Sensing Symp. IGARSS'06, Denver, CO, USA, July 2006.

[7] - "Range Frequency Non-linearity Correction for FMCW SAR," European Patent Application, no. 06076057.6, May 2006.

[8] A. Brancaccio, G. Franceschetti, V. Pascazio, and G. Schirinzi, "Quality Measurements on SC-SAR Images," in Proc. IEEE Int. Geoscience and Remote Sensing Symp. IGARSS'92, 1992, pp. 1149-1151.

[9] P. Franceschetti, M. Tesauro, and P. A. Rosen, "One-bit coding for Spotlight SAR: applications, algorithms and architecture implementation," in Proc. IEEE Int. Geoscience and Remote Sensing Symp. IGARSS'99, 1999, pp. 2273-2274. 

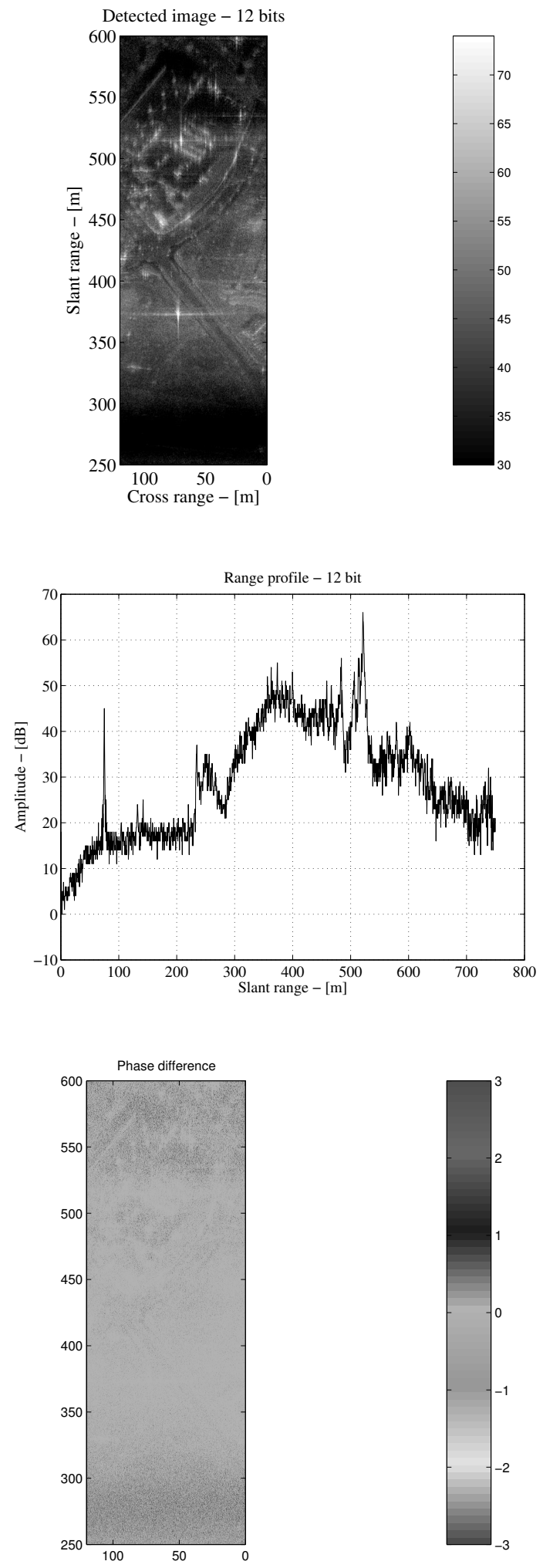
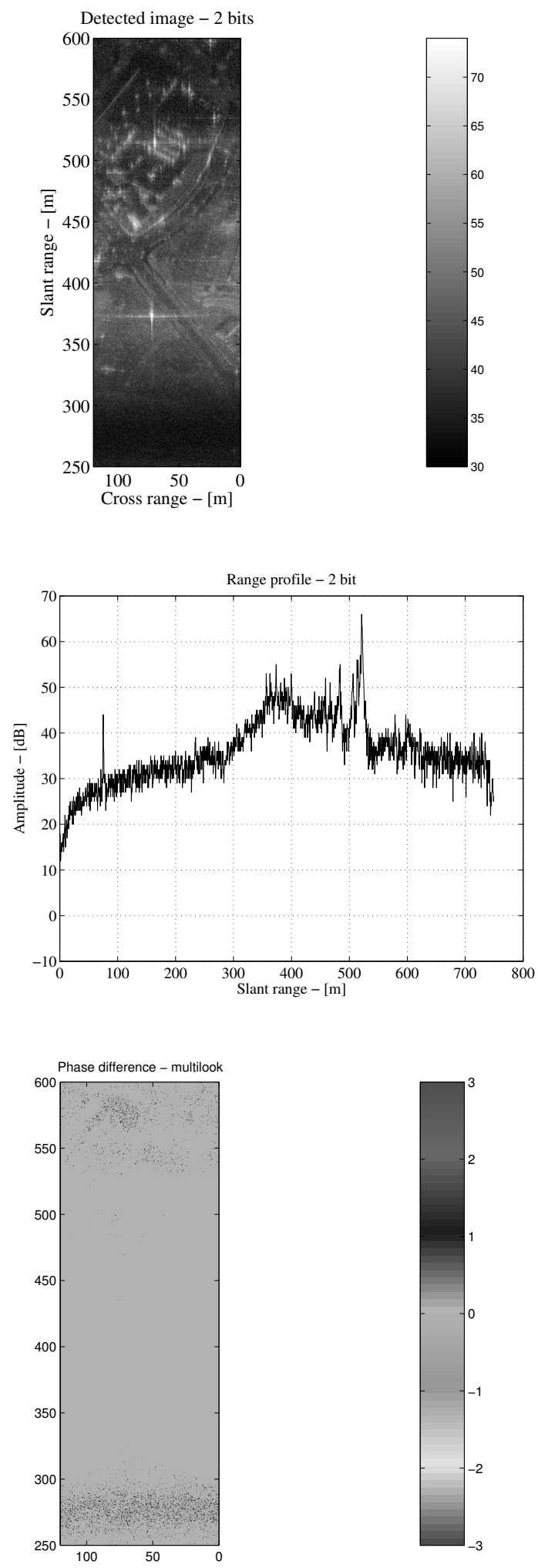

Fig. 3. Processing results. On the first row: intensity image processed starting from 12 bit and 2 bits sampled raw data. On the second row: range profile extracted from the processed images. On the third row: interferogram of the 12 bits and 2 bits (on the left) and then averaging 4 azimuth cells (on the right). 